\title{
Relined fiberglass post: an ex vivo study of the resin cement thickness and dentin-resin interface
}

\section{Niélli Caetano de SOUZA(a) Maurem Leitão MARCONDES(a) Ricardo Vaz BREDA(b) João Batista Blessmann WEBER(a) Eduardo Gonçalves MOTA ${ }^{\text {(a) }}$ Ana Maria SPOHR(a)}

(a) Pontifícia Universidade Católica do Rio Grande do Sul - PUC-RS, School of Dentistry, Department of Restorative Dentistry, Porto Alegre, RS, Brazil.

(b)Pontifícia Universidade Católica do Rio Grande do Sul - PUC-RS, Institute of Biomedical Research, Porto Alegre, RS, Brazil.

Declaration of Interests: The authors certify that they have no commercial or associative interest that represents a conflict of interest in connection with the manuscript.

\section{Corresponding Author:}

Ana Maria Spohr

E-mail:ana.spohr@pucrs.br

DOI: 10.1590/1807-3107BOR-2016.vol30.0077

Submitted: Oct 05, 2015

Accepted for publication: Mar 06, 2016

Last revision: May 3, 2016

\begin{abstract}
The aim of this study was to evaluate the thickness of resin cements in the root thirds when using conventional fiberglass posts $(\mathrm{CP})$ and relined fiberglass posts (RP) in weakened roots and to evaluate the morphological characteristics of the dentin-resin interface. Forty human maxillary anterior teeth had the crown sectioned below the cemento-enamel junction. The canals were endodontically treated and weakened with diamond burs. Teeth were divided into four groups ( $\mathrm{n}=10)$ : Group 1 - CP + RelyX ARC; Group 2 - CP + RelyX U200; Group 3 - RP + RelyX ARC; and Group 4 - RP + RelyX U200. Prior to luting, $0.1 \%$ Fluorescein and $0.1 \%$ Rhodamine B dyes were added to an adhesive and resin cement, respectively. Slices were obtained from the apical, middle, and cervical thirds of the root. Confocal laser scanning microscopy images were recorded in four areas (buccal, lingual, mesial, distal) of each third. In each area, four equidistant measures of the resin cement were made and the mean value was calculated. The interface morphology was observed. The data were submitted to three-way ANOVA and Tukey's test $(\alpha=0.05)$. The interaction between fiberglass posts, resin cement, and root thirds was significant $(p<0.0001)$. The resin cement thicknesses were significantly lower for $\mathrm{RP}$ in comparison with $\mathrm{CP}$, except in the apical third. There was no significant difference between the resin cements for RP. There was formation of resin cement tags and adhesive tags along the root for RP. $\mathrm{RP}$ favored the formation of thin and uniform resin cement films and resin tags in weakened roots.
\end{abstract}

Keywords: Cementation; Resin Cements; Tooth Root.

\section{Introduction}

The use of fiberglass posts for the restoration of endodontically treated teeth is now widespread. There are many advantages to using fiberglass posts compared to metal posts, especially the lower concentration of tensions at the root, which reduces the risk of root fracture. ${ }^{1,2}$ However, adhesion loss is a common failure reported with fiberglass posts, and it usually occurs along the adhesive interface between the root dentin and resin cement. ${ }^{3,4}$

The adhesion to root dentin is hampered by poor visibility, anatomical features, and a high cavity configuration factor. ${ }^{5}$ Fiberglass post retention is influenced by several factors, such as the type and 
shape of the post, the cement, contamination with other chemicals, ${ }^{6}$ the instrumentation techniques and irrigant solutions, ${ }^{7}$ and the thickness of the resin cement. ${ }^{8}$ There is evidence in the literature regarding the effect of the resin cement thickness on the polymerization shrinkage stress. ${ }^{9} \mathrm{~A}$ higher polymerization shrinkage occurring in the resin cement layer can result in increased stress on the dentin-fiberglass post interface, contributing to the formation of structural discontinuities, such as bubbles, cracks, or spaces, and leading to a decreased retention of the fiberglass post. ${ }^{10}$

Fiberglass posts are not always perfectly adapted to the root canals. Thus, one of the techniques proposed for the treatment of large canals is to use a fiberglass post relined with composite resin. ${ }^{11}$ The relined fiberglass post allows for a good adaptation in the root canal, enabling the formation of a thin and uniform layer of resin cement and providing a favorable frictional retention. ${ }^{8}$

Among the factors that determine the degree of post retention, the selection of the luting agent has been widely studied, and resin cements have shown positive results with respect to their mechanical properties and adhesive capacities ${ }^{12,13}$ and are the preferred material to lute the posts. ${ }^{14}$ The self-adhesive resin cements RelyX U100 and RelyX U200 have been shown to have greater bond strength values to root dentin compared with other materials. ${ }^{15,16}$ They are also less technique-sensitive to luting procedures when compared with regular resin cements. ${ }^{17}$ However, resin cements that require an adhesive system are another possibility for luting fiberglass posts. ${ }^{18}$

Considering the relevance of the resin cement thickness and the type of resin cement on fiberglass post retention, the aim of this study was to evaluate the thickness of resin cements in the different root thirds when using relined fiberglass posts (RP) and conventional fiberglass posts $(\mathrm{CP})$ in weakened roots. We also aimed to evaluate the morphological characteristics of the dentin-resin interface through confocal laser scanning microscopy (CLSM). This study was conducted under the null hypothesis that there is no difference in the resin cement thickness among the following variables: a) relined fiberglass posts (RP) and conventional fiberglass posts (CP); b) self-adhesive resin cement and conventional resin cement; and c) cervical, medium, and apical thirds of the root.

\section{Methodology}

Forty human maxillary anterior teeth were obtained from adult patients after approval from the Ethics Committee of the Pontifícia Universidade Católica do Rio Grande do Sul (CAAE 30904114.4.0000.5336). The roots had similar length and similar shape and no curvature in the apical third. After disinfection with $0.5 \%$ chloramine for $48 \mathrm{~h}$, the crowns were removed with a diamond disc (Extec Corp., London, UK) and mounted in a low speed laboratory cutting machine (Labcut 1010, Extec Corp., London, UK) under cooling. The length of the roots was standardized to $15 \mathrm{~mm}$ long.

A step-back preparation technique was used for the endodontic treatment. The teeth were instrumented at a working length of $1 \mathrm{~mm}$ from the apex to a \#55 master apical file (Dentsply/Maillefer, Ballaiques, Switzerland). A step-back technique was performed with stainless-steel K-files \#60 to \#80 (Dentsply/Maillefer, Ballaiques, Switzerland) and Gates Gliden drills \#4 to \#5 (Dentsply/Maillefer, Ballaiques, Switzerland). All enlargement procedures were followed by irrigation with a $2.5 \%$ sodium hypochlorite solution (CSM Chemical Products, Chapecó, Brazil). The prepared root canals were filled with gutta-percha cones (Dentsply, Petrópolis, Brazil) using the lateral condensation technique and Sealer-26 resin sealer (Dentsply, Petrópolis, RJ, Brazil). After the endodontic treatment, the roots were stored in $100 \%$ relative humidity at $37^{\circ} \mathrm{C}$ for $48 \mathrm{~h}$. An endodontist performed the endodontic procedures.

To obtain standardized weakened canals, the coronal gutta-percha was removed with a heated Rhein instrument (Golgran, São Paulo, Brazil) until it reached a length of $10 \mathrm{~mm}$. The canals were enlarged to a length of $10 \mathrm{~mm}$, which was controlled with silicone stops using a Largo drill \#5 (Dentsply/Maillefer, Ballaiques, Switzerland) and high-speed diamond burs \#4138 and \#4137 (KGSorensen, São Paulo, Brazil) with water irrigation. ${ }^{8}$ The roots were embedded in a metallic split cylinder with self-cured acrylic resin (Jet Clássico, São Paulo, Brazil) for stabilization during the luting procedure. 
The roots were randomly divided into four groups $(n=10)$ according to the fiberglass post and resin cement: Group 1(CP ARC) - conventional fiberglass posts no.3 Reforpost (Angelus, Londrina, PR, Brazil) + RelyX ARC (3M, St. Paul, USA); Group 2 (CP U200) - conventional fiberglass post + RelyX U200 (3M, St. Paul, MN, USA); Group 3 (RP ARC) - relined fiberglass post + RelyX ARC; and Group 4 (RP U200) - relined fiberglass post + RelyX U200.

All fiberglass posts were etched with $37 \%$ phosphoric acid for $15 \mathrm{~s}$, followed by silane application (Angelus, Londrina, Brazil). For groups 3 and 4, the bond adhesive Scotchbond Multi-Purpose (3M, St. Paul, USA) was applied and light cured for $20 \mathrm{~s}$ with a light-emitting-diode curing unit (Radii Cal, SDI, Bayswater, Australia) with light intensity of $1.000 \mathrm{~mW} / \mathrm{cm}^{2}$. The posts were then covered with composite resin Z350 (3M, St. Paul, USA) and inserted into root canals that were previously lubricated with hydrosoluble gel (K-Y gel, Johnson \& Johnson, São José dos Campos, Brazil), light cured for $20 \mathrm{~s}$, removed from the canal, and light cured again for $20 \mathrm{~s}$. The luting procedures are described in Table 1.

For the CLSM analysis, $0.1 \%$ fluorescein was incorporated into each bottle of Scotchbond MultiPurpose Plus adhesive system (Activator, Primer and Catalyst) $(40 \mathrm{mg} / \mathrm{mL})$. The dye was mixed directly into the supplied bottle using a mixing device (Vortex
Machine, Scientific Industries, New York, USA) for $2 \mathrm{~h}$ to completely dissolve the dye. $0.1 \%$ Rhodamine B was added to the base resin cement paste and mixed to obtain a paste of uniform shade $(0.32 \mathrm{mg} / \mathrm{mL}) .{ }^{19}$ After luting and storage in water at $37^{\circ} \mathrm{C}$ for $24 \mathrm{~h}$, $1 \mathrm{~mm}$-thick slices were cut from the apical, middle, and cervical thirds of the root using a diamond disc mounted in a low speed laboratory cutting machine under cooling. The cervical third was obtained $1 \mathrm{~mm}$ below the CEJ, the middle third was obtained $4 \mathrm{~mm}$ below the CEJ, and the apical third was obtained $8 \mathrm{~mm}$ below the CEJ. The CLSM images (LSM 5, Zeiss, Jena, Germany) were obtained in dual fluorescence mode using a 20X objective to measure the resin cement thickness and a 63X objective to observe the resin tags. An argon laser at $488 \mathrm{~nm}$ and He-Ne laser at $543 \mathrm{~nm}$ provided excitation energies. The sizes of the recorded images were $187 \times 187 \mu \mathrm{m}^{2}$ with a resolution of 1024 x 1024 pixels.

The images were recorded in four areas (buccal, lingual, mesial, and distal) of each third (apical, middle, cervical) at 20x and analyzed using Image J software S 1.35 (NIH, Bethesda, EUA). The spatial calibration tool was employed to calibrate the program. The measure of the resin cement thickness was made in the four areas (buccal, lingual, mesial, and distal) of each third. In each area, four equidistant measures of the resin cement were made and the mean value

Table 1. Bonding procedures applied in the experimental groups.

\begin{tabular}{|c|c|c|}
\hline Groups & Dentin pre-treatment & Luting agent application \\
\hline G1 - CP ARC & $\begin{array}{l}\text { The canal walls were etched with } 35 \% \text { phosphoric } \\
\text { acid for } 15 \mathrm{~s} \text {, rinsed for } 15 \mathrm{~s} \text {, and gently air-dried. } \\
\text { Excess water was removed from the canal with } \\
\text { absorbent paper points. The Scotchbond Multipurpose } \\
\text { Plus Activator was applied into the root canal with a } \\
\text { microbrush of compatible size and air-dried for } 5 \mathrm{~s} \text {. } \\
\text { Afterward, the Scotchbond Multipurpose Plus Primer, } \\
\text { followed by Catalyst, were applied and air-dried. }\end{array}$ & $\begin{array}{l}\text { The dual-cured resin cement RelyX ARC was mixed and } \\
\text { inserted inside the root canal with a syringe (Centrix, } \\
\text { DFL, Rio de Janeiro, Brazil). The fiberglass post was } \\
\text { inserted into the root canal with light pressure. The } \\
\text { excess luting material was removed and light cured for } \\
40 \text { s on the occlusal surface with a Radii Cal curing } \\
\text { light (SDI, Bayswater, Vic, Australia). }\end{array}$ \\
\hline G2 - CP U200 & $\begin{array}{l}\text { The root canal was rinsed with water. Excess water was } \\
\text { removed from the canal with absorbent paper points. }\end{array}$ & $\begin{array}{l}\text { The mixing tip with endo tip was attached on Rely U200 } \\
\text { Automix syringe. Application of RelyX U200 Automix } \\
\text { cement directly into the root canal. The fiberglass } \\
\text { post was inserted, excess cement was removed, and } \\
\text { the remaining cement cured for } 40 \mathrm{~s} \text { on the occlusal } \\
\text { surface with a Radii curing light. }\end{array}$ \\
\hline G3 - RP ARC & The same as described for $G 1$. & The same as described for $\mathrm{G} 1$. \\
\hline G4 - RP U200 & The same as described for $G 2$. & The same as described for $G 2$. \\
\hline
\end{tabular}

CP: conventional fiberglass post; ARC: RelyX ARC; U200: RelyX U200; RP: relined fiberglass post. 
was calculated. The measure was performed from the RP toward the dentin.

Statistical analysis was performed by applying a three-way analysis of variance (fiberglass posts, resin cements, root thirds) followed by the Tukey post-hoc test at a $95 \%$ confidence level.

\section{Results}

The interaction between fiberglass posts, resin cement, and root thirds was significant $(p<0.0001)$. The resin cement thickness means $(\mu \mathrm{m})$ obtained in the four groups are shown in Table 2.

The resin cement thickness decreased from the apical third to the cervical third when RP was used, regardless of the resin cement (Figures 1A-C). Conversely, the resin cement thickness decreased from the cervical third to the apical third when $\mathrm{CP}$ was used (Figures 2A-C). There was a significant difference between the thirds for both fiberglass posts, regardless of the resin cement $(p<0.05)$.

The resin cement thickness between RP and $C P$ was significantly different in the cervical and middle thirds, with the thickness being significantly smaller with the use of RP $(p<0.05)$. However, there was no significant difference in the apical third, regardless of the resin cement evaluated ( $p>0.05)$. For the RP, there was no significant difference between the resin cement thicknesses at all thirds $(p>0.05)$. A good adaptation of the RP was evident on all images analyzed, and the formation of resin cement tags and adhesive tags in the cervical, middle, and apical thirds was observed when luted with RelyX ARC (Figure 3A). When the RP was luted with RelyX U200, resin cement tags in the cervical and middle thirds were evident (Figure 3B). For the $\mathrm{CP}$, the thicker resin cement was observed in the cervical third when luted with RelyX U200, with complete absence of resin cement penetration in the dentinal tubules. However, evaluating the resin cements in the same root third, there was no significant difference between RelyX U200 and RelyX ARC in the middle and apical thirds ( $p>0.05)$, with a significant difference between the resin cements only occurring for the $\mathrm{CP}$ in the cervical third $(\mathrm{p}<0.05)$. Comparing the images, more bubbles inside the resin cements were observed for the $\mathrm{CP}$.

\section{Discussion}

This study evaluated the thickness of the resin cements RelyX U200 and RelyX ARC in the cervical, middle, and apical thirds when $\mathrm{RP}$ and $\mathrm{CP}$ were luted in weakened roots. The thickness of the resin cements was significantly lower in the RP groups than in the $\mathrm{CP}$ groups in the cervical and middle thirds of the canal, which rejects the first null hypothesis. This finding was expected because the RP adapts better to the canal compared to the $\mathrm{CP}$ once there is less space for the resin cement film. A similar result was found in another study. ${ }^{10}$ However, there was no difference in the resin cement thickness between RP and CP in the apical third of the root. This finding suggests that the apical third is not molded by the RP as efficiently as in the cervical and middle thirds, or that the diameter of the fiberglass post is sufficient to adapt to the apical third because this region has less available space.

The methodology used in the present study to obtain weakened root canals has the advantage of being simple, and it allows the standardization of the procedure in all roots. However, this in vitro simulation does not represent the real clinical situation of weakened roots due to caries and excessiveinstrumentation. In weakened

Table 2. Resin cement thickness means $(\mu \mathrm{m})$ and standard-deviations at cervical, middle and apical thirds using relined fiberglass posts (RP) or conventional fiberglass posts (CP) luted with RelyX U200 (U200) or RelyX ARC (ARC).

\begin{tabular}{lcccc}
\hline & RP $\cup 200$ & CP $\cup 200$ & RP ARC & CP ARC \\
\hline Thirds & $\mathrm{n}=10$ & $\mathrm{n}=10$ & $\mathrm{n}=10$ & $\mathrm{n}=10$ \\
Cervical & $49.85 \pm 9.00^{\mathrm{Cc}}$ & $484.51 \pm 30.00^{\mathrm{Aa}}$ & $40.58 \pm 5.00^{\mathrm{Cc}}$ & $401.61 \pm 28.00^{\mathrm{Ba}}$ \\
Middle & $85.36 \pm 5.00^{\mathrm{Bb}}$ & $320.82 \pm 22.00^{\mathrm{Ab}}$ & $83.42 \pm 5.00^{\mathrm{Bb}}$ & $303.40 \pm 15.00^{\mathrm{Ab}}$ \\
Apical & $125.09 \pm 10.00^{\mathrm{Aa}}$ & $129.81 \pm 17.00^{\mathrm{Ac}}$ & $129.65 \pm 9.00^{\mathrm{Aa}}$ & $127.34 \pm 11.00^{\mathrm{Ac}}$ \\
\hline
\end{tabular}

*Means followed by different capital letters in lines and by different lowercase letters in columns present significant differences according Tukey's test $(\alpha=0.05)$. 
roots, a misfit of the pre-fabricated fiberglass post to the root canal walls can be observed. This misfit happens even using the highest caliber fiberglass post, generating lines of thick cement as observed in this study. The evaluation of the root thirds when using $\mathrm{CP}$ showed the greatest thickness in the cervical and middle thirds, regardless of the resin cement. In addition, more bubbles were observed in both resin cements for $\mathrm{CP}$ in comparison with RP. According to Grandini et al. ${ }^{10}$ a thicker resin cement layer predisposes to blistering, which represents areas of weakness within the material, damaging the bond strength.

The effect of the resin cement thickness on the bond strength of the fiberglass post to the root canal was studied by D'Arcangelo et al. ${ }^{20}$ They found that when the resin cement thickness was too thick, the bond strength was significantly reduced. Similar studies $^{21,22}$ have reported that the resin cement thickness significantly affects the adhesion because an overly thick layer of resin cement is related to greater frequency of adhesion loss between the fiberglass post and the root canal. In addition, the stress of polymerization developed in a thin layer is lower in comparison with a thicker layer.' Several studies have recommended using a thin layer of resin cement to increase the bond strength due to a better micromechanical retention, ${ }^{8,21,22}$ which can be obtained with RP as shown in this study.
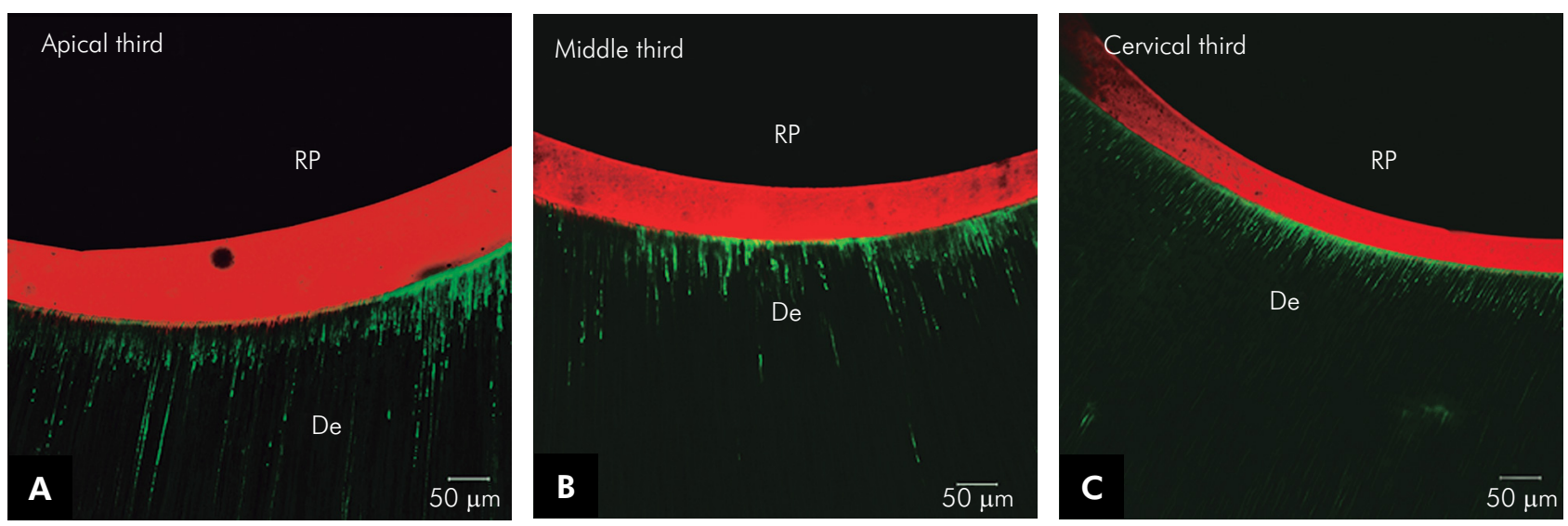

Figure 1. CLSM images (20x) of the interface between relined fiberglass post and root dentin using RelyX ARC (1A, 1B, 1C). Green color: Adper Scotchbond Multi-Purpose Plus Adhesive (activator, primer and catalyst). Red color: resin cement RelyX ARC. The resin cement thickness decreases from the apical third to the cervical third. De: Dentin; RP: Relined fiberglass post.
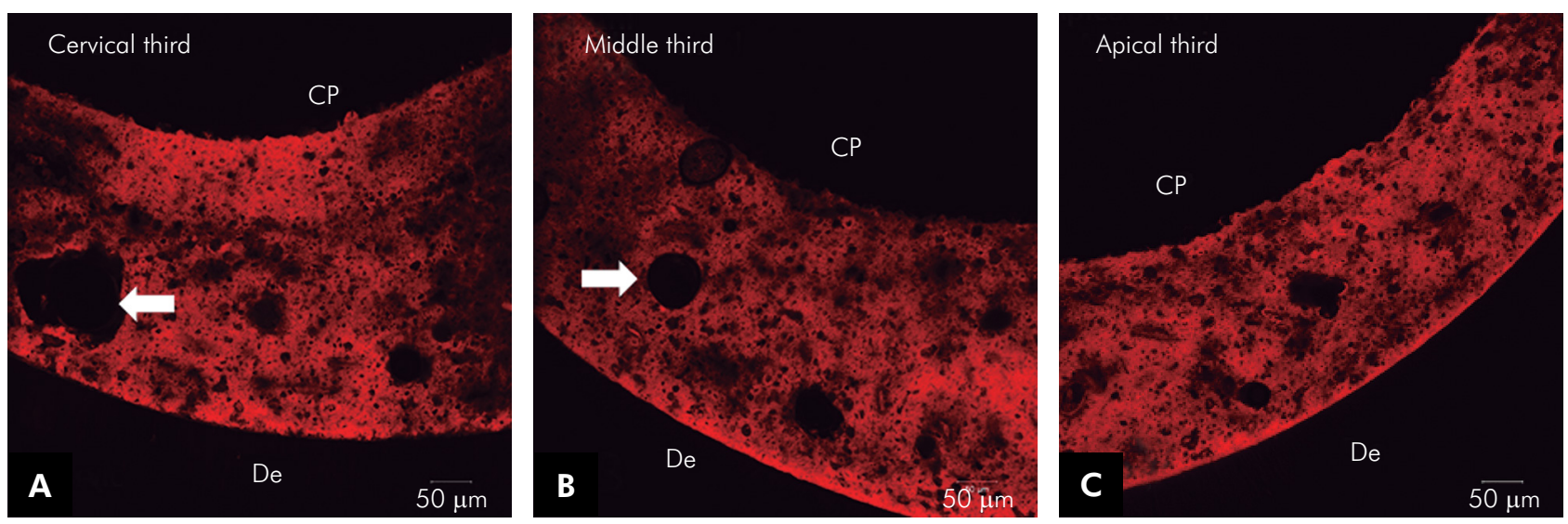

Figure 2. CLSM images (20x) of the interface between conventional fiberglass post and root dentin using RelyX U200 (2A, 2B, 2C). Red color: resin cement RelyX U200. The resin cement thickness decreases from the cervical third to the apical third. De: Dentin; CP: Conventional fiberglass post. Bubbles into the resin cements are identified by the arrows. 

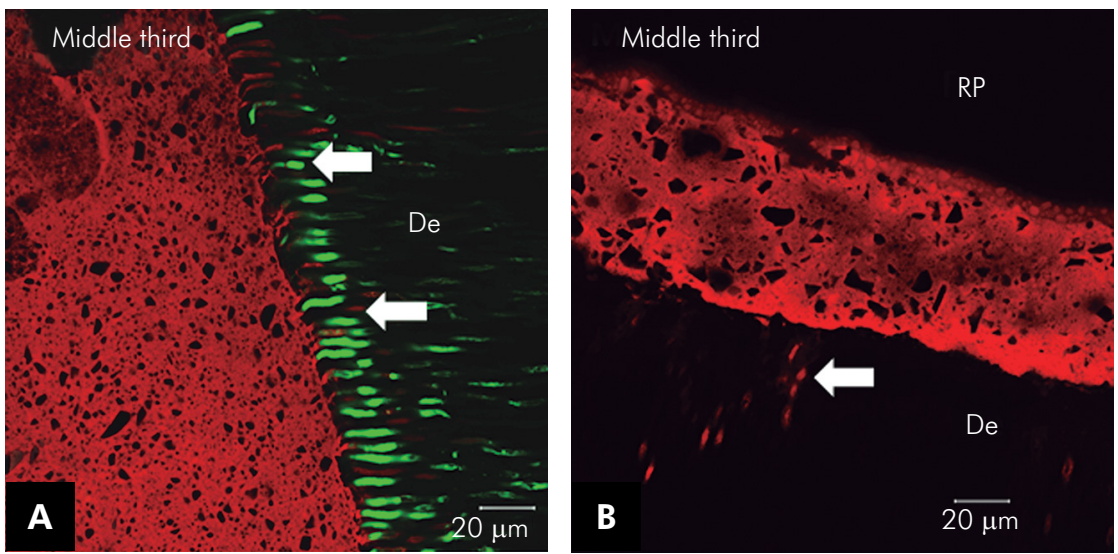

Figure 3. CLSM images (63x) of the interface between the relined fiberglass post and root dentin using RelyX ARC (3A) and RelyX U200 (3B). Green color: Adper Scotchbond Multi-Purpose Plus Adhesive (activator, primer and catalyst). Red color: resin cement. RP: relined fiberglass post; De: dentin. (3A) The presence of tags from both the adhesive system and the resin cement (arrows) are observed. (3B) The image demonstrates the penetration of the RelyX U200 resin cement into the dentinal tubules forming resin tags (arrow).

Most studies have used SEM to evaluate the hybrid layer and the surrounding structures. ${ }^{23,24,25}$ However, technical artifacts can occur during the specimen preparation. ${ }^{26,27}$ CLSM is a method that allows samples to be studied without vacuum in a humid environment. Previous study revealed comparable results convening hybrid layer thickness and penetration into dentinal tubules for SEM and CLSM analyses. ${ }^{28}$ CLSM allows for visualizing different components through the use of dyes. In the present study, two dyes, Rhodamine B and Fluorescein, were used because they have different characteristics. ${ }^{23,29}$ Rhodamine B is a molecule added to the resin cement, and Fluorescein is added to the adhesive system components (activator, primer, and catalyst). ${ }^{30}$ The same proportion $(0.1 \%)$ was used for both dyes. Bitter et al. ${ }^{31}$ showed that Fluorescein inserted into the adhesive showed up without diffusing into the red caused by Rhodamine B in resin cements, marking a clear distinction between the dyes. The effect of the dyes on the polymerization of the materials was studied by D'Alpino et al. ${ }^{32}$ Although fluorescent dyes have the potential to reduce the monomer conversion and the bond strength of the materials to the substrate, the concentration of $0.1 \%$, as was used in the present study, do not influence the polymerization. ${ }^{32}$

The Scotchbond Multipurpose Plus adhesive system was applied before the luting with RelyX ARC. The technique consists of etching the root canal with $37 \%$ phosphoric acid, followed by application of the activator, primer, and catalyst to make the adhesive a dual material, allowing for self-cure polymerization in regions not irradiated by the LED unit. ${ }^{19}$ Because there are several operative steps for using this adhesive system, there is a greater technical sensitivity. To facilitate the luting procedure, manufacturers have developed self-adhesive resin cements, which do not require the prior application of an adhesive system.

The null hypothesis that conventional resin cements and self-adhesive resin cements do not influence the thickness of the resin cement was partially rejected. The thickness of RelyX U200 was higher than RelyX ARC only for CP in the cervical third. The cervical third is the largest region of the root canal to be filled by the resin cement. It is possible that the resin cement RelyX U200, which has a higher viscosity than RelyX ARC, could more adequately fill the cervical third, providing a greater thickness of resin cement. In the RP groups, there was no significant difference in the three thirds for the two resin cements. The RP adapts to the root canal and exerts pressure on the resin cement against the canal walls, favoring the penetration of the resin cements into the substrate. Although there was no significant difference for the thickness of the resin cements in most root thirds analyzed, a trend for lower values for RelyX ARC was observed, except for the apical third for RP. This finding is probably related to the adhesive system that is applied, which 
forms a film thickness that decreases the space to be filled by the resin cement RelyX ARC.

The third null hypothesis was rejected because there was a significant difference between the thirds of the root canal for both fiberglass posts. For RP, the resin cement thickness decreased from apical third to the cervical third, and the opposite occurred for CP. This finding suggests that the RP has better ability to mold the root canal in the cervical third compared to the middle and apical thirds.

One of the most interesting findings of this study was that resin cement tags were observed in the cervical and middle thirds when RP was luted with RelyX U200. Due to the thixotropic behavior of RelyX U200, the application of pressure decreases its viscosity and improves its adaptation to the cavity walls, ${ }^{33}$ allowing resin tag formation. In addition, this improved adaptation can decrease air bubbles inside the resin cement and optimize the physical interactions with the dental substrate through Van der Waals forces, hydrogen bonds, and charge transfers. ${ }^{34}$ This favors the micromechanical retention within the dentinal tubules by the resin cement tags. For RelyX $\mathrm{ARC}$, the penetration of both the adhesive and the resin cement into the dentinal tubules for RP was observed forming adhesive tags and resin cement tags. Because polymerization takes place only when the resin cement comes in contact with the adhesive inside the canal, the luting pressure generated by the

\section{References}

1. Clavijo VGR, JMSN Reis, Kabbach W, Silva ALF, Oliveira Júnior OB, Andrade MF. Fracture strength of flared bovine roots restored with different intraradicular posts. J Appl Oral Sci. 2009;17(6):574-8. doi:10.1590/S1678-77572009000600007

2. Schmitter M, Hamadi K, Rammelsberg P. Survival of two post systems: five-year results of a randomized clinical trial. Quintessence Int. 2011;42(10):843-50.

3. Monticelli F, Grandini S, Goracci C, Ferrari M. Clinical behavior of translucent-fiber posts: a 2-year prospective study. Int J Prosthodont. 2003;16(6):593-6.

4. Ferrari M, Cagidiaco MC, Grandini S, De Sanctis M, Goracci C. Post placement affects survival of endodontically treated premolars. J Dent Res. 2007;86(8):729-34. doi:10.1177/154405910708600808

5. Naumann M, Koelpin M, Beuer F, Meyer-Lueckel H.10-year survival evaluation for glass-fiber-supported
$\mathrm{RP}$ pushes the resin cement and the adhesive against the walls of the canal, favoring penetration into the dentinal tubules. It is proposed that the resin cement tags can contribute to the retention of the RP, and consequently in an increased pull-out bond strength compared with $\mathrm{CP}{ }^{8}$

\section{Conclusions}

Within the limitations of the present study, it could be concluded that

a. The thickness of resin cement is lower with relined fiberglass posts compared to conventional fiberglass posts in the cervical and middle thirds, with no difference in the apical third;

b. The resin cement RelyX U200 was thicker than RelyX ARC only in the cervical third using the conventional fiberglass post;

c. The thickness of resin cement decreased from the apical third to the cervical third with relined fiberglass posts; the opposite occurred with the conventional fiberglass posts;

d. Relined fiberglass posts favored the formation of resin cement tags along the root canal.

\section{Acknowledgments}

The authors thank the Institute of Biomedical Research of PUCRS for the images of confocal laser scanning microscopy.

postendodontic restoration: a prospective observational clinical study. J Endod. 2012;38(4):432-5. doi:10.1016/j.joen.2012.01.003

6. Stockton LW. Factors affecting retention of post systems: a literature review. J Prosthet Dent. 1999;81(4):380-5. doi:10.1016/S0022-3913(99)80002-X

7. Santana FR, Soares CJ, Silva JÁ, Alencar AH, Renovato SR, Lopes LG, Estrela C. Effect of instrumentation techniques, irrigant solutions and artificial accelerated aging on fiberglass post bond strength to intraradicular dentin. J Contemp Dent Pract. 2015;16(7):523-30. doi:10.5005/jp-journals-10024-1716

8. Macedo VC, Silva ALF, Martins RM. Effect of cement type, relining procedure, and length of cementation on pull-out bond strength of fiber posts. J Endod. 2010;36(9):1543-6. doi:10.1016/j.joen.2010.04.014 
9. Jongsma LA, Ir NJ, Kleverlaan CJ, Feilzer AJ. Reduced contraction stress formation obtained by a two-step cementation procedure for fiber posts. Dent Mater. 2011;27(7):670-6. doi:10.1016/j.dental.2011.03.008

10. Grandini S, Goracci C, Monticelli F, Borracchini A, Ferrari M. SEM evaluation of the cement layer thickness after luting two different posts. J Adhes Dent. 2005;7(3):235-40.

11. Grandini S, Sapio S, Simonetti M. Use of anatomic post and core for reconstructing an endodontically treated tooth: a case report. J Adhes Dent. 2003;5(3):243-7.

12. Gaston BA, West LA., Liewehr FR, Fernandes C, Pashley $\mathrm{DH}$. Evaluation of regional bond strength of resin cement to endodontic surfaces. J Endod. 2001;27(5):321-4. doi:10.1097/00004770-200105000-00001

13. Bouillaguet S, Troesch S, Wataha JC, Krejci I, Meyer JM, Pashley DH. Microtensile bond strength between adhesive cements and root canal dentin. Dent Mater. 2003;19(3):199-205. doi:10.1016/S0109-5641(02)00030-1

14. Sarkis-Onofre R, Pereira-Cenci T, Opdam NJ, Demarco FF. Preference for using posts to restore endodontically treated teeth: findings from a survey with dentists. Braz Oral Res. 2015;29(1):1-6. doi 10.1590/1807-3107BOR-2015.vol29.0001

15. Amaral M, Rippe MP, Bergoli CD, Monaco C, Valandro LF. Multi-step adhesive cementation versus one-step adhesive cementation: push-out bond strength between fiber post and root dentin before and after mechanical cycling. Gen Dent. 2011; 59(5):e185-91.

16. Nova V, Karygianni L, Altenburger MJ, Wolkewitz M, Kielbassa AM, Wrbas KT. Pull-out bond strength of a fibre-reinforced composite post system luted with self-adhesive resin cements. J Dent. 2013;41(11):1020-6. doi:10.1016/j.jdent.2013.08.011

17. Skupien JA, Sarkis-Onofre R, Cenci MS, Moraes RR, Pereira-Cenci T. A systematic review of factors associated with the retention of glass fiber posts. Braz Oral Res. 2015;29. pii: S1806-83242015000100401. doi:10.1590/1807-3107BOR-2015.vol29.0074

18. Souza ACO, Gonçalves FCP, Anami LC, Melo RM, Bottino MA, Valandro LF. Influence of insertion techniques for resin cement and mechanical cycling on the bond strength between fiber posts and root dentin. J Adhes Dent. 2015;17(2):175-80. doi:10.3290/j.jad.a33993

19. Arrais CA, Miyake K, Rueggeberg FA, Pashley DH, Giannini M. Micromorphology of resin/dentin interfaces using 4 th and 5 th generation dual-curing adhesive/cement systems: a confocal laser scanning microscope analysis. J Adhes Dent. 2009;11(1):15-26.

20. D’Arcangelo C, D’Amario M, Vadini M, Zazzeroni S, De Angelis F, Caputi S. An evaluation of luting agente application technique effect on fibre post retention. J Dent. 2008;36(4):235-40. doi:10.1016/j.jdent.2008.01.004

21. Özcan E, Çetin AR, Tunçdemir AR, Ülker M. The effect of luting cement thicknesses on the push-out bond strength of the fiber posts. Acta Odontol Scand. 2013;71(3-4):703-9. doi:10.3109/00016357.2012.715194
22. Egilmez F, Ergun G, Cekic-Nagas I, Vallittu PK, Lassila LV. Influence of cement thickness on the bond strength of tooth-colored posts to root dentin after thermal cycling. Acta Odontol Scand. 2013;71(1):175-82. doi:10.3109/00016357.2011.654257

23. Wang Y, Spencer P. Exploring the nature of acid-resistant hybrid layer with wet bonding. Oper Dent. 2004;29(6):650-5.

24. Abou-Id LR, Morgan LFSA, Silva GAB, Poleto LTA, Lanza LD. Ultrastructural evaluation of the hybrid layer after cementation of fiber posts using adhesive systems with different curing modes. Braz Dent J. 2012;23(2):116-21. doi:10.1590/S0103-64402012000200005

25. Soares CJ, Pereira JC, Valdivia ADCM, Novais VR, Meneses MS. Influence of resin cement and post configuration on bond strength to root dentine. Int Endod J. 2012;45(2):136-45. doi:10.1111/j.1365-2591.2011.01953.x

26. Van Meerbeek B, Vargas M, Inoue S, Yoshida Y, Perdigão J, Lambrechts $\mathrm{P}$ et al. Microscopy investigations: techniques, results, limitations. Am J Dent. 2000;13(Spec No):3D-18D.

27. Pereira CNB, Daleprane B, Barbosa PF, Moreira AN, Magalhães CS. Qualitative evaluation of scanning electron microscopy methods in a study of the resin cement/dentine adhesive interface. Microsc Microanal. 2014;20(1):268-75. doi:10.1017/S143192761301369X

28. Bitter K, Paris S, Mueller J, Neumann K, Kielbassa AM. Correlation of scanning electron and confocal laser scanning microscopic analyses for visualization of dentin/adhesive interfaces of the root canal. J Adhes Dent. 2009;11(1):7-14.

29. Bitter K, Gläser C, Neumann K, Blunck U, Frankenberger R. Analysis of resin-dentin interface morphology and bond strength evaluation of core materials for one stage post-endodontic restorations. PLoS One. 2014;9(2):e86294. doi:10.1371/journal.pone.0086294

30. Aguiar TR, Andre CB, Arrais CAG, Bedran-Russo AK, Giannini M. Micromorphology of resin-dentin interfaces using self-adhesive and conventional resin cements: a confocal laser and scanning electron microscope analysis. Int J Adhes Adhes. 2012t;38:69-74. doi:10.1016/j.ijadhadh.2012.05.009

31. Bitter K, Paris S, Pfuertner C, Neumann K, Kielbassa AM. Morphological and bond strength evaluation of different resin cements to root dentin. Eur J Oral Sci. 2009;117(3):326-33. doi:10.1111/j.1600-0722.2009.00623.x

32. D'Alpino PH, Pereira JC, Svizero NR, Rueggeberg FA, Pashley $\mathrm{DH}$. Factors affecting use of fluorescent agents in identification of resin-based polymers. J Adhes Dent. 2006;8(5):285-92.

33. De Munck J, Vargas M, Van Landuyt K, Hikita K, Lambrechts P, Van Meerbeek B. Bonding of an auto-adhesive luting material to enamel and dentin. Dent Mater. 2004;20(10):963-71. doi:10.1016/j.dental.2004.03.002

34. Goracci C, Cury AH, Cantoro A, Papacchini F, Tay FR, Ferrari M. Microtensile bond strength and interfacial properties of self-etching and self-adhesive resin cements used to lute composite onlays under different seating forces. J Adhes Dent. 2006;8(5):327-35. 